\title{
What are the health and well-being impacts of community gardening for adults and children: a mixed method systematic review protocol
}

\author{
Rebecca Lovell ${ }^{1 *}$, Kerryn Husk ${ }^{1}$, Alison Bethel ${ }^{2}$ and Ruth Garside ${ }^{1}$
}

\begin{abstract}
Background: Community gardening is defined by its shared nature; gardeners work collectively to manage a garden for shared benefit. Although communal gardening activities, and recognition of their perceived benefits have a long history, it is in recent years that interest has developed in assessing the potential of the approach to address many of the threats to health and wellbeing faced by global populations. Community gardening may address chronic and non-communicable disease through the provision of opportunities for physical activity, improved nutrition and reduced stress. Participation in the gardening activities may improve wellbeing through increased social contact, culturally valued activities and mitigation of food poverty. The benefits of community gardening are argued to extend beyond the participants themselves through more coherent and cohesive communities, improved physical environments and the sharing of the products of the labour. While there are many claims made and an emerging body of research, no previous systematic review has sought to identify and synthesise the evidence in a global context.
\end{abstract}

Methods: The objectives of the mixed method systematic review are to understand the health and wellbeing impacts of active participation in community gardening. Both quantitative and qualitative evidence will be sought using a broad and diverse search strategy to address the four review questions:

1) does active involvement in community gardening lead to improved health or wellbeing;

2) if so, how does active involvement in community gardening affect health and wellbeing;

3) are there different impacts for different population groups (for instance according to age, socio-economic status or sex); and

4) do different types of community gardening (for example producing vegetables or a flower garden) or in different contexts have different types of impacts?

A theoretical framework, informed by an initial theory of change model, will illustrate the outcomes of participation and any mechanisms of action (i.e. how such impacts are achieved). The synthesis will be sensitive to factors which may affect the impacts, such as the context of the activities, the demographics of participants, and the implementation and specifics of the community gardening interventions.

Keywords: Community garden, Health, Wellbeing, Quality of life, Volunteer, Mixed method

\footnotetext{
*Correspondence: r.lovell@exeter.ac.uk

${ }^{1}$ European Centre for Environment and Human Health, University of Exeter

Medical School, University of Exeter, Truro TR1 3HD, UK

Full list of author information is available at the end of the article
} 


\section{Background}

\section{Community gardens}

A community garden is defined and described by the American Community Garden Association (the ACGA) as a place

that can be urban, suburban, or rural. It can grow flowers, vegetables or community. It can be one community plot, or can be many individual plots. It can be at a school, hospital, or in a neighborhood. It can also be a series of plots dedicated to "urban agriculture" where the produce is grown for a market [1].

The UK focused Federation of City farms and Community Gardens notes that community gardens are diverse in size and focus; they range from tiny wildlife gardens to fruit and vegetable plots on housing estates, to large community polytunnels [2]. The activity is described using many different terms, including community-agriculture, -farms or -market gardens, however for the remainder of this protocol the term 'community garden' will be used to refer to this family of related activities.

Communal gardening activity has a long history, with evidence of 2000 year old communally managed plots in Cornwall, in the south-west of the UK, to the re-emergence of the idea during both World Wars where small plots, called 'Victory Gardens', were given over to the coproduction of food [3]. In the 1960's and 70's concerns about urban decay, community breakdown and issues of food quality prompted a re-emergence of the concept [4]. More recently 'community gardening' has become a globally popular activity; networks and coordinating groups have been identified in the USA, UK, southern Africa, and Australia, and evidence of community gardening can be found elsewhere including Asia and South America.

Community gardening is generally a non-profit making endeavour, though in some case profits are made through the sale of surplus products (often the explicit aim of projects in the developing world) or where members run courses in gardening and other related skills. While some gardens are developed and used as settings for health or wellbeing focused interventions - for instance as therapeutic setting for people suffering from dementia, mental health issues and social isolation [5-12] - it is likely the majority do not have this as a stated objective.

\section{Communities and the gardens}

For the purposes of this review, 'communities' are defined as groups of individuals, either by geographical proximity or brought together as interest groups, who work together to develop and maintain a garden as a shared resource.

A fundamental aspect of community gardening is that it is a shared endeavour, often from the very conception of the project to the management and maintenance of the garden. The gardens can be located on public or private land and may be managed (possibly by a paid coordinator) by an external organisation (such as a charity or land trust) or by the gardeners themselves. Participants range from those keen to improve the aesthetic of their neighbourhoods to those who perceive community gardens to be a more sustainable source of fruit and vegetables. Some participants are involved for social or health reasons. Participants will on the whole be volunteers; this is one of the factors which differentiates community gardening from similar endeavours such as (some forms of) market gardening where gardeners may receive a salary. The size of the 'community' involved in the project also varies hugely, from the garden sharing projects common in the UK (where, for example, elderly people unable to manage their large gardens share the spaces with local families, who in turn share their produce with the landowner) to the much larger scale market-garden projects aiming to achieve a consistent supply of fruit and vegetable to their members and beyond.

The aims of the garden typically focus on providing opportunities for local residents, improving local environments and/or on producing fruit and vegetables for food. Community gardening is defined as distinct, at least for the purposes of this review protocol, from individual or family based residential or allotment gardening, and from city farms (where they only manage livestock) and other collective livestock management. Urban agriculture has been the subject of a separate review; however the authors focused solely on low and middle income countries [13].

\section{Community gardening and health and wellbeing outcomes}

Populations around the world face a range of threats to health and wellbeing which could potentially be addressed by initiatives such as community gardening $[14,15]$. These threats include the non-communicable diseases and their contributory risk factors such as low levels of physical activity and poor diet. Poor mental health is projected to be one of the most extensive population health issues and current trends, such as the increase in factors such as loneliness and poor social contact, will further exacerbate this situation [16,17]. Poverty and food insecurity also contribute to the burden of poor health [18]. More broadly environmental degradation (both social and physical) has also been shown to impact on health and wellbeing [19].

Community gardening is a complex multi-factorial activity, likely to have multiple impacts (direct and indirect) on the health and wellbeing of those taking part $[14,15]$. These impacts may be positive but also, potentially, negative. The specific ways in which community gardening may impact on health and wellbeing are numerous and operate on a variety of scales and through several mechanisms (see Figure 1). Community gardening has been 


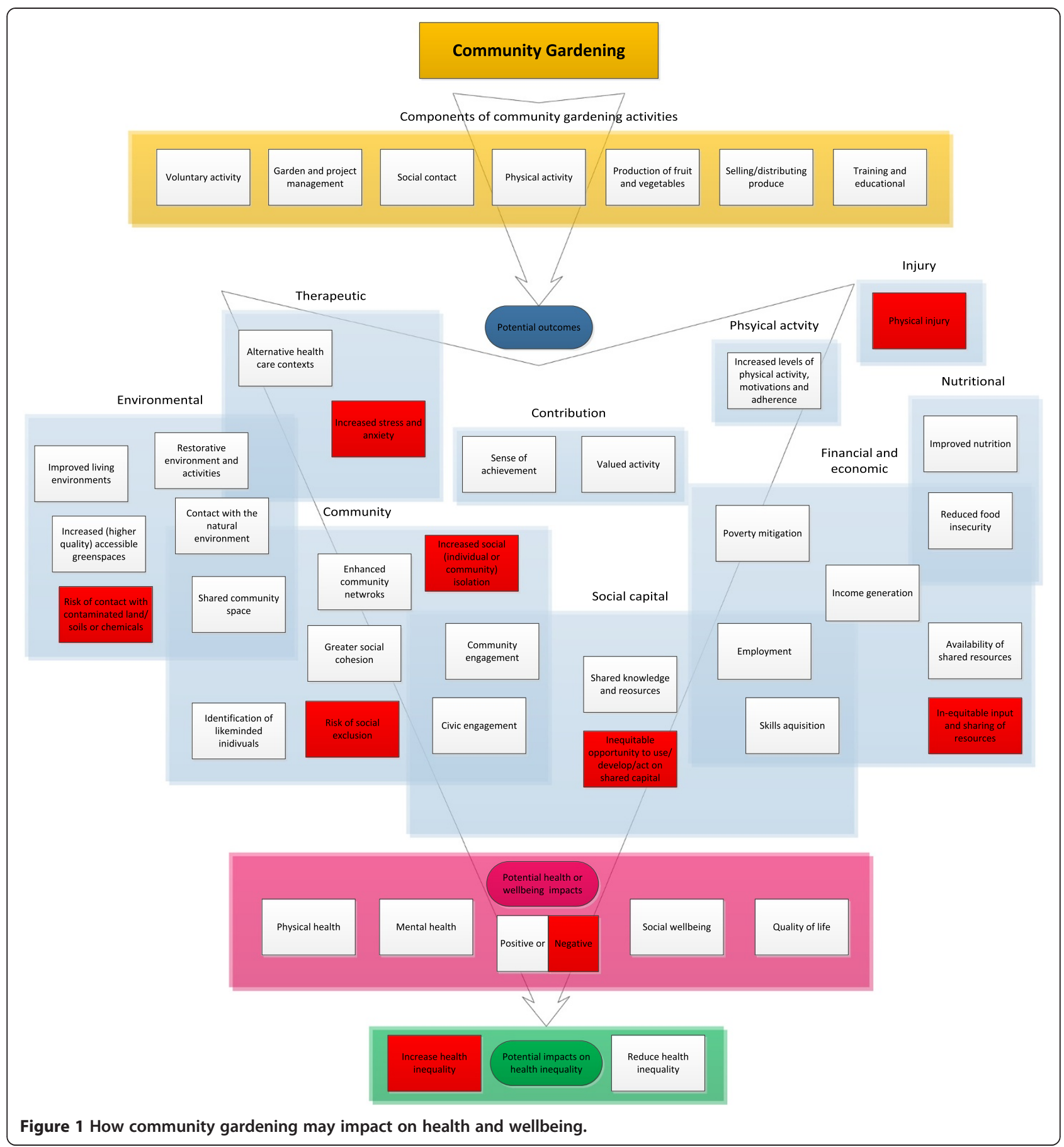

argued to have potential to improve the nutritional status of those involved. For example, where community gardeners focus on fruit and vegetable production there is the potential that participants could improve their diets through more positive perceptions towards, awareness of, and access to these foodstuffs [20-22]. Community gardening may also be effective in mitigating the effects of food insecurity and poverty both for those undertaking the gardening activities but also for the wider community where the produce may be distributed or sold [21,23]. Regular involvement in gardening may contribute to achieving recommended levels of physical activity, a wellestablished feature of healthy life styles [5,24]. Furthermore the communal, collective nature of the activities within these projects may promote adherence and greater motivations to continuing higher levels of physical activity [5]. Some have argued that community gardening may have a role in reducing stress and promoting better mental 
health [25-27]. This is thought to come about through various mechanisms but includes time spent in neutral spaces (i.e. those not associated with specific institutions) and the therapeutic nature of the activities [28]. Positive engagement in worthwhile activities, such as volunteering for community benefit, may promote a sense of value and achievement, both are factors which contribute to enhanced sense of quality of life (QOL) [29,30].

Developing and strengthening community and social ties through, for example, providing opportunities for positive social contact between community members who share common interests may be a further way in which community gardening promotes better health and wellbeing [31]. Community gardening may also promote good health and wellbeing by strengthening social resilience and motivating the implementation of other neighbourhood improvements, especially in deprived areas [32,33]. Evidence from South America suggests that involvement in community gardening leads to increased civic engagement, thereby promoting social wellbeing and QOL [26,34,35]. For example, through community gardening members may need to engage with the planning and legal processes associated with land ownership and management [36]. More widely, features of community gardening such as environmental education, skills acquisition and access to shared resources may contribute to QOL and aspects of personal and social capital [37]. Wellbeing is also strongly linked with employment and income, and community gardening may lead to the development of skills suitable to enhance employability [38]. Finally contact with the natural world has been argued to be of benefit to health and wellbeing by providing opportunities for mental restoration and stress recovery [39].

It should also be noted that there may be potential for community gardening to negatively impact on health and wellbeing. For instance the programme may be exclusionary, with specific members of the community being unable (through, for example, lack of resources whether financial, material or in terms of social capital) or desiring to take part. There is the potential that community gardening may exacerbate or increase local health inequalities and individual or group level social exclusion [40]. Furthermore there are physical risks of the gardening activities such as injury or over exertion. Disturbance and inadvertent consumption of metals and other contaminants in the ground may cause serious health damage [41]. The stress associated with managing the project and people involved may also result in negative health and wellbeing outcomes [42].

There are several theoretical understandings as to how community gardening, in common with several other forms of community engagement activity, might promote better health and wellbeing. Examples include the development of the concept of 'social or community capital', community development, and sustainability (in terms of food and nutrition and in reference to community structures) [43-45].
Furthermore the suite of theories, such as Biophilia and Attention Restoration, which seek to explain the apparent benefit from contact with the natural environment $[46,47]$, may also be of value in understanding if and how community gardening might impact on wellbeing.

An initial exploratory theory of change model has been developed by the authors using evidence derived from the scoping reviews for this protocol and from previous research [30]. The model illustrates the processes though which community gardening activities could lead to the kinds of outcomes (both positive and negative (red boxes)), such as contact with the natural environment, mitigation of poverty, or improved nutrition, which may have direct or indirect impacts to the health and wellbeing of those taking part (Figure 1) [48].

\section{Reviewing the evidence relating to community gardening} Whilst there appears to be a considerable body of literature which has sought to understand the impacts of community gardening, no previous comprehensive systematic review which has reliably addressed the potential health and wellbeing benefits of community gardening was identified. Several linked reviews, some of which were not carried out using systematic review methodologies, were identified but these were limited in scope (e.g. focusing on one geographical area, such as the UK [15]), considered just one of the potential outcomes (e.g. nutrition [49]) or a specific population (e.g. amongst youth [50]). The results of previous reviews are indicative that there is a sizable body of research and that community gardening may have beneficial impacts to health and wellbeing [15]. Furthermore, there is evidence that policy makers and those interested in cost-effective health improvement programmes around the world are increasingly considering supporting this type of activity [51]. It is therefore timely that the evidence of effectiveness is reviewed in a systematic and rigorous manner. The findings of the scoping further justify the necessity of a more comprehensive review.

A related review of the impacts of school based gardening is being undertaken in conjunction with this review and with input from the authors. This review is titled "A systematic review of the health and well-being impacts of school gardening" and focuses solely on the impacts of communal gardening activities in the school setting to school children, school staff, family and community members (all ages) [52]. Therefore the present review will exclude all studies relating to the impacts of community gardens in school settings.

\section{Objectives of the review}

The objectives of this mixed method (including both quantitative and qualitative evidence) review are to assess the health and well-being impacts. 
The objectives of this mixed method (including both quantitative and qualitative evidence) review are to assess the health and well-being impacts experienced by adults and/or children following active participation in community gardening in any context (other than school gardening). The aims are to provide further understanding by answering the following research questions:

1. Does active involvement in community gardening lead to improved health and/or wellbeing (e.g. physical, mental, social or QOL)?

2. If so, how does active involvement in community gardening affect health and/or wellbeing?

3. Are there different impacts for different population groups (for instance according to age, socio-economic status or sex)?

4. Do different types of community gardening (for example producing vegetables or a flower garden) or in different contexts (e.g. urban/rural, developed/low-middle income country, community/ therapeutic setting) have different types of impacts?

The empirical evidence relating to the above questions, both quantitative and qualitative, will be identified, appraised and synthesized. Evidence drawn from quantitative studies will be used to appraise the strength and direction of effect of community gardening on health and wellbeing outcomes. The qualitative evidence will be used to understand the experience and meaning of participation in community gardening, the various pathways to engagement, process of involvement and factors which may mediate experiences. A theoretical framework, informed by our initial theory of change model, will be produced which will aim to illustrate the outcomes of participation and any mechanisms of action $[30,48]$. The synthesis will be sensitive to factors which may affect the impacts, such as the context of the activities, the demographics of participants, and the implementation and specifics of the community gardening interventions. This review will consider potential mechanisms of action primarily through research q2 but also by addressing formal academic theories (where available) and through the lay or practitioner theories used in existing evaluations.

\section{Stakeholders}

A project advisory group will be convened to guide the process and focus of the review. Individuals who have experience of community gardening, associated funding, relevant research and of effective dissemination of envi ronment-health evidence to policy makers and practitioners will be invited to take part. It is anticipated that the advisory group will be drawn from experts based in the UK, while it is appreciated this may introduce a bias this is for pragmatic reasons relating to resources. If potential advisory group members are identified in other parts of the world, they will be invited to participate and contribute their ideas and suggestions via email or teleconferencing.

\section{Methods}

The review team will draw on the systematic review methodology promoted by the Collaboration for Environmental Evidence [53] in conjunction with that endorsed by the Cochrane Collaboration [54].

\section{Searches}

Informal scoping searches (conducted spring 2014) have indicated that there are a good number of relevant, includable studies that have been published and indexed in formal academic databases (databases searched for scoping included PubMed, Scopus, Web of Science, Greenfile and Enviroment Complete) and that a considerable number will also be found in the grey literature. A previous review of environmental enhancement activities [30] highlighted the value of allocating a significant proportion of time and resources to searching these non-traditional sources. Therefore a suite of search techniques will be used including academic database searches, citation chasing (forward and backward), web-searches, grey literature searches of organisational web sites, contact with key authors and organisations. Primary searches (of academic databases) will be designed and undertaken by an information specialist (AB). Secondary searches of grey literature and specialist, coordinating organisations will be led by $\mathrm{RL}$ and $\mathrm{KH}$.

\section{Search terms and strategy}

The key search terms have been derived from scoping searches and through a reading of the literature. The project reference group will also advise the review team on potential terminology. The individual terms (as listed below in 'Medline search string', lines 1-24) have been incorporated (lines 25-29) into a primary string for Medline and will be adapted as appropriate for each database. Searches will be conducted on title, abstract and key words (.tw):

Medline search string:

1 exp Gardening/

2 Garden*.tw.

3 Horticultur*.tw.

4 Agricultur*.tw.

5 Allotment*.tw.

6 Greening.tw.

7 Cultivat*.tw.

8 Propagat*.tw.

9 Food product".tw.

10 Vegetable*.tw.

11 Rrural.tw. 
12 Suburban*.tw.

13 Communit*.tw.

14 Communal.tw.

15 Shared.tw.

16 Collective*.tw.

17 Neighbourhood*.tw.

18 Neighborhood*.tw.

19 Guerrilla.tw.

20 Rebel.tw.

21 Co-operative ${ }^{*}$. tw.

22 Urban*.tw.

23 Therapeutic*.tw.

24 Pop-up*.tw.

252 or 3 or 4 or 5 or 6 or 7 or 8 or 9 or 10

2611 or 12 or 13 or 14 or 15 or 16 or 17 or

18 or 19 or 20 or 21 or 22 or 23 or 24

27 ((Garden* or Horticultur* or Agricultur* or

Allotment* or Greening or Cultivat* or Propagat* or

Food product* or Vegetable*) adj3 (Rural or

Suburban* or Communit* or Communal or Shared

or Collective* or Neighbourhood* or Neighborhood*

or Guerrilla or Rebel or Co-operative* or Urban* or

Therapeutic* or Pop-up*)).tw.

281 and 26

2927 or 28

The search strategy, developed in conjunction with the information specialist (AB), will use appropriate search syntax and will be modified for use in multiple health and social science electronic databases:

- AMED on EBSCOHost

- ASSIA on ProQuest

- CINAHL on EBSCOHost

- BNI on ProQuest

- EMBASE on OvidSP

- HMIC on OvidSP

- Web of Knowledge

- MEDLINE on OvidSP

- PsycINFO on OvidSP

- Social Policy and Practice on OVIDSP

- EThOS via the British Library

- OpenGrey

A year limit of 1990 will be applied. The scoping searches found very little published evidence relating to community gardening previous to 1990, for instance Web of Science (WoS) identified just one paper published prior to 1990 which was found to be un-includable (see Figure 2). There will be no limiters applied according to study design. No language limit will be applied. The searches will be conducted in English (it is recognised that this may introduce a language bias to the results, however the limit is again due to pragmatic use of resources).
The scoping searches of non-academic sources including organisational websites identified a number of studies that are unpublished meaning they are unlikely to be indexed in academic databases; therefore an extensive grey literature search will be conducted. The process will be looping and iterative as it necessarily relies on the identification of includable studies to progress the searches. The methods used will include:

- Forwards and backwards citation searching of all includable studies, both manually and using tools such as Web of Knowledge;

- Identification and searching of the web presence of community gardens and umbrella organisations such as the Federation of City Farms and Community Gardens in the UK and the American Community Garden Association in the US, comparable organisations will be sought for low and middle income countries;

- Contacting, via email, telephone and web searches of the above organisations to request studies or suggestions for sources of evidence (including internal library catalogues not available online);

- Searches of grey literature indexes such as Open Grey (http://www.opengrey.eu/) (conducted with database searches);

- Searches of academic web engines such as Google scholar;

- Web searches of Google, Bing etc.;

- Searches of relevant academic repositories;

- Hand searching of key academic and organisational journals and publications (e.g. Ecohealth, Environment and Society, Royal Horticultural Society journal) consulting with the project reference group for suggestions of studies, researchers and other potential contacts.

The search terms that will be used in the grey literature searches will include appropriate combinations of (Garden* or Horticultur* or Agricultur* or Allotment* or Greening or Cultivat*) and (Communit* or Communal or Shared or Collective* or Neighbourhood* or Neighborhood* or Cooperative*). When searching websites, academic web engines such as Google Scholar and other digital grey literature sources, the first 100 hits will be searched. If a high proportion of the first 100 hits (e.g. $>10 \%$ ) prove to be potentially includable the results will be further searched until no more references are found. Where only a small number of hits are returned $(<100)$ the whole results list will be searched.

Citation searches for key papers including those listed below will be carried out in databases such as Web of Knowledge: 


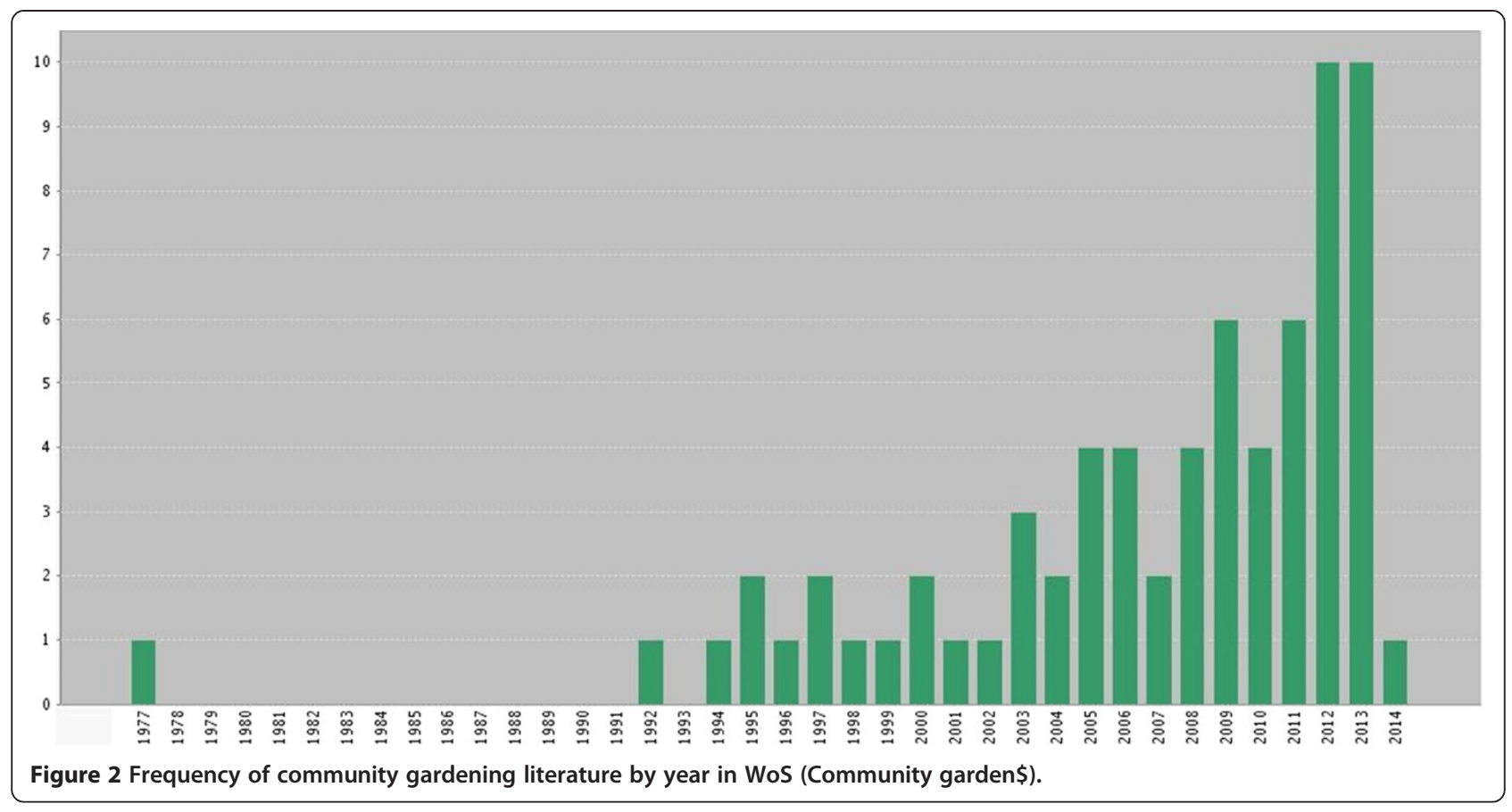

- Austin, E. N., Johnston, Y. A. M., \& Morgan, L. L. (2006). Community Gardening in a Senior Center: A Therapeutic Intervention to Improve the Health of Older Adults Therapeutic Recreation Journal 40(1).

- Barnidge, E. K., Hipp, P. R., Estlund, A., Duggan, K., Barnhart, K. J., \& Brownson, R. C. (2013).

Association between community garden participation and fruit and vegetable consumption in rural Missouri. The International Journal Of Behavioral Nutrition And Physical Activity, 10(1), 128-128.

- Blair, D., Giesecke, C. C., \& Sherman, S. (1991). A dietary, social and economic evaluation of the Philadelphia urban gardening project. Journal of Nutrition Education, 23(4), 161-167.

- Carney, P. A., Hamada, J. L., Rdesinski, R., Sprager, L., Nichols, K. R., Liu, B. Y., et al. (2012). Impact of a community gardening project on vegetable intake, food security and family relationships: a communitybased participatory research study. J Community Health, 37(4), 874-881.

- Gonzalez, M. T., Hartig, T., Patil, G. G., Martinsen, E. W., \& Kirkevold, M. (2010). Therapeutic horticulture in clinical depression: a prospective study of active components. J Adv Nurs, 66(9), 2002-2013.

- Heim, S., Stang, J., \& Ireland, M. (2009). A garden pilot project enhances fruit and vegetable consumption among children. J Am Diet Assoc, 109 (7), 1220-1226.

- Kingsley, J. Y., Townsend, M., \& Henderson-Wilson, C. (2009). Cultivating health and wellbeing:
Members' perceptions of the health benefits of a Port Melbourne community garden. Leisure Studies, 28(2), 207-219.

- Weltin, A. M., \& Lavin, R. P. (2012). The effect of a community garden on HgA1c in diabetics of Marshallese descent. J Community Health Nurs, 29(1), 12-24.

- Zick, C. D., Smith, K. R., Kowaleski-Jones, L., Uno, C., \& Merrill, B. J. (2013). Harvesting more than vegetables: the potential weight control benefits of community gardening. Am J Public Health, 103(6), $1110-1115$.

Search results will be exported to EndNote 2011 (Thompson Reuters). Searches will be recorded using the Preferred Reporting Items for Systematic Reviews meta-Analysis (PRISMA) guidelines [55].

\section{Inclusion and exclusion criteria Types of interventions}

Active and first-hand gardening activities (e.g. growing flowers, re-greening, horticulture, market gardening/ farming) which take place in any 'garden' which is managed and used by a group of people in common (i.e. linked by locality/neighbourhood/interest) will be included. Flower gardens, vegetable gardens (including community allotments) and wildlife gardens will be included. Although the duration of participation in the activities will not determine inclusion in the review, where possible it will be considered as a potential subgroup factor. 
Includable community gardening activities are listed below but are not expected to be exhaustive:

- garden creation

- garden maintenance

- weeding

- planting

- propagation and cultivation

- producing flowers, fruit or vegetables

- harvesting

The review will also include a group of activities which are commonly described as 'therapeutic' gardening and horticulture. These are activities which, like community gardening, take place in a shared garden space but in which the purpose of the activities is specifically related to recuperation, healing and restoration of the participants. The synthesis will be sensitive to the differences between those community garden activities which have explicit health and wellbeing aims and those that do not. It is likely that such differences will necessitate the aims being treated as a sub-group factor.

There are a range of terms used to describe associated community gardening activities and places: for instance, some forms of guerilla gardening, collective horticulture, pocket park creation, and community greening. These will all be includable provided they meet the basic definition of communal gardening activities as described above. Where possible the potential differential impacts of activities undertaken in different contexts (e.g. urban/rural, or flower/ vegetable gardens) or according to different motivations (e.g. therapeutic/leisure-interest based) will be examined.

Where studies are identified in which a range of activities (but including community gardening) are assessed only those studies where the outcomes relate solely or in the majority (i.e. more than $50 \%$ of the activity) to gardening can be extracted will be included. All decisions relating to these types of studies will be recorded and justified in the final report.

Private use of gardens, parks, and school gardens will be excluded as these are considered to be fundamentally different activities or settings. In the case of school gardens the impacts of 'community' gardening in this setting will be addressed in a separate review [52]. This review will not consider impacts to the environment, for example increases in biodiversity.

The description of the intervention above is not exhaustive, the expert advisory board will provide further guidance on the breadth and intentions of community gardening activities. The search strategy will be modified if necessary.

\section{Types of participants}

Adults or children (as defined in each includable study) actively participating in community gardening, as defined in previous sections, in any setting other than the school. Our analysis will be sensitive to potential differential impacts on specific subgroups of people; this will be carried out according to factors such as socio-economic status, employment, age, and physical, mental or sensory health.

It is anticipated that includable studies may relate to both voluntary and 'non-voluntary' (e.g. those on probationary schemes, prison or psychiatric unit gardens or those for whom choice is at least constrained, i.e. work programmes associated with benefits provision) participants. Studies, or the aspects of a study focusing on the impact of community gardening activities which form part or all of a paid role will be excluded.

The routes to participation (e.g. motivations, pressures, barriers) will be considered during analysis to further understand how community gardening could impact on the health and wellbeing of participants and in what situations and contexts. It is anticipated that the qualitative evidence will be used to examine the routes to participation in community gardening.

\section{Study types \\ Quantitative studies}

Following a review in a similar area it is anticipated that there will be few if any randomised, controlled studies in this field [30]. A broad range of study designs will, therefore, be included and our full report will critically reflect on the implications this might have for the robustness of the findings.

The following types of quantitative studies will be considered for inclusion in the review (definitions of includable study designs are taken from the UK's National Institute of Health and Care Excellence's guide to study designs [56]:

- Randomised controlled trials (RCTs), cluster RCTs

- Quasi-RCTs, cluster quasi-RCTs

- Controlled before-and-after studies with any appropriate comparator group (i.e. not involved in community gardening)

- Interrupted time series; the intervention points must be clearly defined with three outcome measurements times (e.g. before, during and after participation in the gardening activities)

- Case-control studies; these will be included where the selection and comparability criteria are clear and where steps have been taken to reduce bias. Baseline characteristics should have been comprehensively reported to allow for assessment of potential confounders.

If there are no higher order study designs available, uncontrolled before and after studies (uBAs) will be considered. To be includable the uBA subject selection criteria and method of inclusion/exclusion to the uBA 
should be well defined, as should attrition rates and reasons. It is recognised that uBAs cannot assess causality and are subject to multiple sources of bias, however this approach to study inclusion has been used in several previous reviews of subjects where studies using higher order deigns are not common [30].

In studies using a controlled study design any appropriate comparator activity will be considered, an example would be voluntary activity in a community library or no activity.

\section{Qualitative studies}

Qualitative research, from any discipline or theoretical tradition that uses recognised qualitative methods of data collection and analysis, and which aims to describe the experiences of those taking part in community gardening will be considered for inclusion. Data collection methods may include (but will not be limited to): focus groups, individual interviews, ethnographic interviews, participant or systematic observation, documentary analysis, or audio visual/note collection. Methods of analysis may include: grounded theory, narrative analysis, thematic analysis, hermeneutic phenomenological analysis, discourse analysis. To be considered for inclusion qualitative studies should have adequately described methodologies and have presented results using $1^{\text {st }}$ or $2^{\text {nd }}$ order constructs with adequate supportive evidence in the form of direct quotes, diary entries etc.

Mixed studies: Studies making use of both quantitative and qualitative aspects within the design will also be includable if one or both elements meet the methodological criteria for the respective approach as detailed above.

\section{Outcomes}

\section{Quantitative research}

Includable primary outcomes will include any recognised measure of health and well-being, whether physical or mental (including emotional and quality of life), assessed using self-reported and objective measures. It is anticipated that these will include:

- Physiological outcomes (for example, heart rate, cortisol levels, per cent body fat or BMI assessed using standardised tools).

- Physical health outcomes, general or specific (assessed using, for example, objective tools such as accelerometers, validated measures such as elements of the Short Form Health Survey or using other selfreported measures of health status, general function or capacity).

- Mental and emotional health outcomes (these may be assessed using validated scales such as the Warwick-Edinburgh Mental Well-being Scale or using measures of emotional response or attention restoration/stress recovery).

- Quality of life outcomes (these may be assessed using, for example, the Short Form (36 or 12) quality of life measures or the Euroquol EQ-5D measures).

Additional outcomes, including adverse or unintended outcomes and mechanisms known to be determinants of health or wellbeing (e.g. physical activity), will be considered where reported alongside health/well-being outcomes:

- Physical activity behaviours (for example, frequency, pattern and intensity of activity, physical activity beliefs and intentions).

- Cognitive performance.

- Rate of recovery from illness or disability (physical or mental).

- Recording of positive feelings, or the meaning ascribed by participants (whether the participant enjoyed/liked the experience).

- Data on outcomes related to social cohesion (e.g. UK Citizenship survey 2009-10, cohesion measures), where reported.

- Dietary information.

Adverse or unintended outcomes will also be included, these may include:

- Physical injury.

- Decline in mental and emotional health status.

- Failure to recover from illness or injury.

- Increased stress.

\section{Qualitative research}

Includable qualitative study's findings will be in the form of themes, concepts and metaphors relating to the experience, meaning and perceived impacts of community gardening and any factors that help or hinder their success.

\section{Potential effect modifiers and heterogeneity}

It is anticipated that there will be considerable heterogeneity in research design, method, population, outcomes and analysis. This is likely to be due to factors such as the nature of the activities to the disciplinary origins of studies. In addition heterogeneity is expected to be related to the breadth of the aims and uses of the activities that will be potentially includable in the review, which will range from traditional community gardens developed to provide vegetables through to specific therapeutic interventions.

Therefore only where there is sufficient homogeneity between designs, methods, populations and outcomes will full meta-analysis be undertaken. Meta-analysis will 
be used to inform wider conclusions regarding the impacts of the activities if there is sufficiently limited heterogeneity. Chi-Square tests will indicate heterogeneity and caution will be taken when applied to small sized studies.

As it is not possible to anticipate the nature and extent of the data available the potential effect modifiers cannot be identified a-priori. However it is expected that, if data allows, the impact of factors such as age, gender, length of engagement with intervention, and type, location and context of intervention will be considered; this is not an exhaustive list but indicative of the factors which may be relevant.

\section{Study selection}

Titles and abstracts (where available) identified through the search process (both academic and grey literature) will be uploaded into EndNote, de-duplicated and double screened against the inclusion criteria by 2 reviewers (RL, $\mathrm{KH}$ ), and disagreements resolved through discussion or, where required, a third reviewer (RG). Potentially includable studies will be obtained in full text then double screen by two reviewers (RL and $\mathrm{KH}$ ) with disagreement resolved through discussion or with reference to the opinion of a third reviewer (RG).

\section{Data extraction and management}

Data will be extracted into bespoke forms developed specifically for this review by two researchers (RL and KH).

For both quantitative and qualitative studies details regarding the intervention (frequency, locations, nature of activities, theory etc.), participants (number, demographics etc.) and researcher/evaluators (funding, institution etc.) will be recorded. For quantitative studies specifics regarding study design and execution will be extracted, these will include: when outcomes were assessed, nature of controlling activities, allocation of participants to intervention or control groups, and potential confounders. Specific data extracted from qualitative studies will include themes, concepts (first and second order concepts will be clearly identified), and quotes identified by the authors of the studies.

Equity data will also be extracted according to the PROGRESS-Plus factors [57].

\section{Assessment of the risk of bias and study quality}

Appraisal of the quality and risk of bias of both quantitative and qualitative studies will be undertaken by two reviewers (RL and $\mathrm{KH})$, and disagreements resolved through discussion and, where necessary, a third reviewer (RG). The quality and any risk of biases will be considered during synthesis and where necessary included in reporting of the results.

\section{Quantitative studies}

The quality and risk of bias in quantitative studies will be assessed using the Effective Public Health Practice Project tool [58]. The tool considers factors such as risk of selection bias, completion rates, study design, confounders, blinding, intervention integrity, and analytical robustness. If meta-analyses are undertaken publication biases will be explored using funnel plots. Any potential sources of bias will be fully considered and discussed in the report. Where insufficient detail or missing data is found this will be requested from study authors. Where sets are missing data meta-analyses will be conducted in accordance with intention to treat analysis. If further data are not available or obtainable, analyses will be conducted but limitations and extent of missing data will be noted and highlighted in the report.

\section{Qualitative studies}

The quality of the conduct and reporting of the included qualitative studies will be assessed using the Wallace criteria [59]. This approach considers theoretical perspective, question, study design, context, sampling, data collection, analysis, reflexivity, generalisability and ethics.

There is the potential that there will be certain biases associated with the studies considering community gardening activities. In particular it is likely that the origin of the research, which in some cases may not have academic origins or have been peer-reviewed, may introduce a bias. The experience of the authors in undertaking previous reviews of similar activities suggest that a number of the studies may have been conducted by those delivering the intervention, this may represent a further source of bias. It is also likely that there will be significant shortcomings in the quality of reporting (of method, intervention specifics and results). Additionally, funding for studies will also potentially come from organisations with a vested interest in community gardening and study authors may also be employed by these organisations.

\section{Data synthesis and presentation \\ Quantitative data}

Quantitative studies will be used to appraise the strength and direction of evidence of effect and, if possible, to produce more precise estimates of effect through random effects meta-analyses. Where the quantitative study design or outcomes are so heterogeneous as to preclude metaanalysis a narrative synthesis approach will be used [60].

Continuous data will be reported, where possible, using the original scale, in the form of means and standard deviations (SD). Scales will be combined where appropriate and standardised mean differences (SMDs) calculated. Ordinal data (in the form of validated measurement scales), are also likely and in line with guidelines by the Cochrane Collaboration [61]; shorter scales will be grouped and 
analysed as dichotomous outcomes for which odds ratios will be calculated. For longer ordinal scales, counts and rates will treated in the analyses as continuous. Where outcome measures in all studies are on the same scale, weighted mean differences will be calculated. Where appropriate forest plots will be produced through the meta-analyses.

Repeated observations and re-occurring events are likely in this review, where these are present consecutive analyses will be performed relating to each time-frame. Particular attention will be paid to ensuring that participants are only included once in meta-analyses. It is not anticipated that cluster RCTs will be identified for inclusion in this review, however where these are included analysis will be performed using a summary statistics for each cluster. Similarly, where identified and included multi-arm studies will be analysed using single pairwise comparisons calculated by combining groups.

Where data allow (i.e. sufficient homogeneity of study design, intervention, outcome, measures) formal metaanalysis in the form of random effects models will be produced. Heterogeneity of studies will be assessed using the $1^{2}$ statistics where possible, qualitatively where not, and meta-analyses will be performed where combination would produce meaningful results. Where significant heterogeneity is present, quantitative studies will be combined and explored using narrative synthesis approaches (for example, using tabulation and juxtaposition of results according to factors associated with the heterogeneity).

Where possible visual techniques will be used to present the quantitative synthesis in an accessible manner, for example if meta-analysis is undertaken forest or harvest plots will be created. If using a narrative approach tables (indicating factors such as study quality, study $\mathrm{n}$, strength and direction/s of results) will be used to visually represent the trends in the results.

\section{Qualitative data}

Qualitative studies will be used to capture the holistic experience and meaning of community gardens for those involved and to understand the pathways to engagement, process of involvement and factors which may mediate their experiences. Exact methods of synthesis for the included qualitative research will depend on the nature of the evidence identified. For example, where data are largely descriptive, thematic analysis will be used, whereas more conceptually rich studies may be better suited to a meta-ethnographic approach $[62,63]$.

As with the quantitative data visual techniques will be used to illustrate the nature of the qualitative data and synthesis.

\section{Overarching synthesis}

The individual quantitative and qualitative syntheses will be conducted in parallel and then brought together in an overarching narrative synthesis (Popay et al. [60]). Narrative synthesis allows for the contextualised integration of diverse forms of evidence to better understand the topic of the review. This approach is particularly useful in reviews of complex intervention effectiveness such as community gardening. If data allows, the analysis will be sensitive to impacts on different groups of people (e.g. age, adults, and children, those with mental ill health or learning disabilities, those with dementia, those recovering from specific conditions or addictions) or according to programme type (e.g. location, activity type, aim etc.). The qualitative evidence will also be used to examine those factors which help or hinder the successful development, use and sustainability of the particular form of community gardens for different groups of individuals. The combined narrative synthesis will be used to develop the theory of change and conceptual model (see Figure 1) [48]. The theory of change developed through this review will be informed by academic, practitioner and lay theories evident in the evidence; this will then be incorporated into and illustrated in the conceptual model. The model will be grounded in and built from the synthesised results of both the quantitative and qualitative evidence.

\begin{abstract}
Abbreviations
AB: Alison Bethel; KH: Kerryn Husk; OD: Odds ratio; PRISMA: Preferred reporting Items for systematic reviews meta-analysis; QOL: Quality of life; RG: Ruth Garside; RL: Rebecca Lovell; SF36/12: Short form health survey; RCT: Random controlled trial; SD: Standard deviation; SMD: Standardised mean differences; WoS: Web of Science; UBA: Uncontrolled before and after study.
\end{abstract}

\section{Competing interests}

Rebecca Lovell is involved in the health and wellbeing committee of the Wildlife Gardening Forum http://www.naturalengland.org.uk/advice/ wildlifegardening/defaultaspx. The authors $\mathrm{KH}, \mathrm{AB}$ and RG declare that they have no competing interests.

\section{Authors' contributions}

$R G$ conceived of the review. RL and $K H$ authored the protocol with $R G$ and $\mathrm{Ab}$ reviewing each iteration. $\mathrm{AB}$ in collaboration with $\mathrm{RL}, \mathrm{KH}$ and $\mathrm{RG}$ devised the search strategy. All authors contributed to the completion of the protocol draft.

\section{Authors' information}

Rebecca Lovell, Kerryn Husk, Ruth Garside: European Centre for Environment and Human Health, University of Exeter Medical School, University of Exeter, Truro, UK, TR1 3HD.

Alison Bethel: Peninsula Technology Assessment Group, Veysey Building, Salmon Pool Lane, Exeter UK, EX2 4SG.

\section{Acknowledgements}

The European Centre for Environment and Human Health (part of the University of Exeter Medical School) is in part financed by the European Regional Development Fund Programme 2007 to 2013 and European Social Fund Convergence Programme for Cornwall and the Isles of Scilly. Thanks are due to Heather Ohly and Rachel Wigglesworth for their input during the initial development of this protocol.

\section{Author details}

'European Centre for Environment and Human Health, University of Exeter Medical School, University of Exeter, Truro TR1 3HD, UK. ${ }^{2}$ NIHR CLAHRC South West Peninsula (PenCLAHRC), Veysey Building, Salmon Pool Lane, Exeter EX2 4SG, UK. 
Received: 16 May 2014 Accepted: 6 August 2014

Published: 7 October 2014

\section{References}

1. What is community gardening. https://communitygarden.org/resources/.

2. Community gardens. www.farmgarden.org.uk/farms-gardens.

3. Mobayed G: Community gardens: a place for cultivating social and community development. Sydney: The University of New South Wales; 2009

4. Oral History Project. http://www.farmgarden.org.uk/farms-gardens/oralhistory-project.

5. Austin EN, Johnston YAM, Morgan LL: Community gardening in a senior center: a therapeutic intervention to improve the health of older adults. Thera Rec J 2006, 40:48-56.

6. Bwika RA: Community gardening practices, motivations, experiences, perceived health effects and policy. Vancouver: University of British Columbia; 2011.

7. Detweiler MB, Sharma T, Detweiler JG, Murphy PF, Lane S, Carman J, Chudhary AS, Halling MH, Kim KY: What is the evidence to support the use of therapeutic gardens for the elderly? Psychiatry Invest 2012, 9:100-110.

8. Ennis I: Sacred groves of the city: a look at contemplative gardens in New Zealand. In Expanding roles for horticulture in improving human well-being and life quality. Edited by Relf D. Toronto: Acta Horticulturae; 2004:113-120

9. Eriksson $\mathrm{T}$, Westerberg $\mathrm{Y}$, Jonsson $\mathrm{H}$ : Experiences of women with stressrelated ill health in a therapeutic gardening program. Can J Occup Ther 2011, 78:273-281.

10. Lis-Balchin M: The therapeutic garden. J R Soc Promot Health 2001, 121:64.

11. Milligan C, Gatrell A, Bingley A: 'Cultivating health': therapeutic landscapes and older people in northern England. Soc Sci Med 2004, 58:1781-1793.

12. Rens $M$, de Jacomine L, Connie K, Natascha P: Develop a sustainable 'therapeutic' garden for frail older persons. J Clin Nurs 2010, 19:75.

13. Stewart R, Korth M, Langer L, Rafferty S, Da Silva N, Rooyen C: What are the impacts of urban agriculture programs on food security in low and middle-income countries? Environ Evid 2013, 2:7.

14. Draper C, Freedman D: Review and analysis of the benefits, purposes, and motivations associated with community gardening in the United States. J Community Pract 2010, 18:458-492.

15. Guitart D, Pickering $C$, Byrne J: Past results and future directions in urban community gardens research. Urban For Urban Green 2012, 11:364-373.

16. Ferrari AJ, Charlson FJ, Norman RE, Patten SB, Freedman G, Murray CJL, Vos T, Whiteford HA: Burden of depressive disorders by country, sex, age, and year: findings from the global burden of disease study 2010. PLOS Med 2013, 10:e1001547.

17. Steptoe A, Shankar A, Demakakos P, Wardle J: Social isolation, loneliness, and all-cause mortality in older men and women. Proc Natl Acad Sci 2013 , 110:5797-5801.

18. Ramsey R, Giskes K, Turrell G, Gallegos D: Food insecurity among adults residing in disadvantaged urban areas: potential health and dietary consequences. Public Health Nutr 2012, 15:227-237.

19. Speldewinde $P C$, Cook A, Davies $P$, Weinstein $P$ : A relationship between environmental degradation and mental health in rural Western Australia. Health Place 2009, 15:880-887.

20. Alaimo K, Packnett E, Miles RA, Kruger DJ: Fruit and vegetable intake among urban community gardeners. J Nutr Educ Behav 2008, 40:94-101.

21. Barnidge EK, Hipp PR, Estlund A, Duggan K, Barnhart K, Brownson RC: Association between community garden participation and fruit and vegetable consumption in rural Missouri. Int J Behav Nutri Physic Activ 2013, 10:128.

22. Lautenschlager $L$, Smith C: Understanding gardening and dietary habits among youth garden program participants using the theory of planned behavior. Appetite 2007, 49:122-130.

23. Hanna AK, Oh P: Rethinking urban poverty: a look at community gardens. Bull Sci Technol Soc 2000, 20:207-216.

24. Beaglehole R, Bonita R, Horton R, Adams C, Alleyne G, Asaria P, Baugh V, Bekedam H, Billo N, Casswell S, Cecchini M, Colagiuri R, Colagiuri S, Collins T, Ebrahim S, Engelgau M, Galea G, Gaziano T, Geneau R, Haines A, Hospedales J, Jha P, Keeling A, Leeder S, Lincoln P, McKee M, Mackay J, Magnusson R, Moodie R, Mwatsama M, et al: Priority actions for the non-communicable disease crisis. Lancet 2011, 377:1438-1447.

25. Blake A, Cloutier-Fisher D: Backyard bounty: exploring the benefits and challenges of backyard garden sharing projects. Local Environ 2009, 14:797-807.
26. Wakefield S, Yeudall F, Taron C, Reynolds J, Skinner A: Growing urban health: community gardening in South-East Toronto. Health Promot Int 2007, 22:92-101.

27. Ohmer ML, Meadowcroft P, Freed K, Lewis E: Community gardening and community development: individual, social and community benefits of a community conservation program. J Community Pract 2009, 17:377-399.

28. Eriksson $T$, Karlstrom $E$, Jonsson $H$, Tham $K$ : An exploratory study of the rehabilitation process of people with stress-related disorders. Scand J Occup Ther 2010, 17:29-39.

29. Hayashi N, Wada T, Hirai H, Miyake T, Matsuura Y, Shimizu N, Kurooka H, Horiuchi S: The effects of horticultural activity in a community garden on mood changes. Environ Control Biol 2008, 46:233-240.

30. Husk K, Lovell R, Cooper C, Garside R: Participation in environmental enhancement and conservation activities for health and well-being in adults. John Wiley \& Sons, Ltd: In Cochrane Database of Systematic Reviews; 2013

31. Agustina I, Beilin R: Community gardens: space for interactions and adaptations. Procedia Soc BehavSci 2012, 36:439-448.

32. Armstrong D: A survey of community gardens in upstate New York: implications for health promotion and community development. Health Place 2000, 6:319-327.

33. Okvat HA, Zautra AJ: Community gardening: a parsimonious path to individual, community, and environmental resilience. Am J Community Psychol 2011, 47:374-387.

34. Saldivar-Tanaka L, Krasny ME: Culturing community development, neighborhood open space, and civic agriculture: the case of Latino community gardens in New York City. Agri Human Values 2004, 21:399-412.

35. Shinew KJ, Glover TD, Parry DC: Leisure spaces as potential sites for interracial interaction: community gardens in urban areas. J Leis Res 2004, 36:336-355.

36. Henderson BR, Hartsfield $\mathrm{K}$ : Is getting into the community garden business a good way to engage citizens in local government? Nat/ Civ Rev 2009, 98:12-17.

37. Krasny ME, Tidball KG: Community gardens as contexts for science, stewardship, and civic action learning. Cities and the Environment (CATE) 2009, 2:8.

38. Hill A: A helping hand and many green thumbs: local government, citizens and the growth of a community-based food economy. Local Environ 2011, 16:539-553.

39. Bowler D: The importance of nature for health: is there a specific benefit of contact with green space? In Systematic review - collaboration for environmental evidence. Bangor; UK: Collaboration for Environmental Evidence; 2010.

40. Parry DC, Glover TD, Shinew KJ: 'Mary, mary quite contrary, how does your garden grow?': examining gender roles and relations in community gardens. Leis Stud 2005, 24:177-192.

41. Bugdalski L, Lemke LD, McElmurry SP: Spatial variation of soil lead in an urban community garden: implications for risk-based sampling. Risk Anal 2013, 34:17-27.

42. Schmelzkopf K: Urban community gardens as contested space. Geogr Rev 1995, 85:364-381.

43. Kawachi I, Kennedy BP, Glass R: Social capital and self-rated health: a contextual analysis. Am J Public Health 1999, 89:1187-1193.

44. Woolcock M, Narayan D: Social capital: Implications for development theory, research, and policy. The World Bank Research Observer 2000, 15:225-249.

45. Stedman RC: Sense of place as an indicator of community sustainability. For Chron 1999, 75:765-770.

46. Kaplan R, Kaplan S: The experience of nature: a psychological perspective. Cambridge: Cambridge University Press; 1989.

47. Kellert S, Wilson EO: The biophillia hypothesis. Washington: Island Press; 1995.

48. Anderson LM, Petticrew M, Rehfuess E, Armstrong R, Ueffing E, Baker $P$, Francis $D$, Tugwell $P$ : Using logic models to capture complexity in systematic reviews. Res Synthesis Methods 2011, 2:33-42.

49. Pomerleau J, Lock K, Knai C, McKee M: Interventions designed to increase adult fruit and vegetable intake can be effective: a systematic review of the literature. J Nutr 2005, 135:2486-2495.

50. Robinson-O'Brien R, Story M, Heim S: Impact of garden-based youth nutrition intervention programs: a review. J Am Diet Assoc 2009, 109:273-280.

51. Community Gardens. http://www.cdc.gov/healthyplaces/healthtopics/ healthyfood/community.htm. 
52. Ohly H, Wigglesworth R, Bethel A, Husk K, Lovell R, Garside R: A systematic review of the health and well-being impacts of school gardening. In PROSPERO international prospective register of systematic reviews. York: York Centre for Reviews and Dissemination; 2014.

53. Collaboration for Environmental Evidence. http://www.environmentalevi dence.org/.

54. The Cochrane Collaboration. http://www.cochrane.org/.

55. Moher D, Liberati A, Tetzlaff J, Altman DG, The PG: Preferred reporting items for systematic reviews and meta-analyses: the PRISMA statement. PLoS Med 2009, 6:e1000097.

56. National Institute for Health and Clinical Guidance: Methods for the development of NICE public health guidance (second edition). London: NHS; 2009.

57. Welch V, Petticrew M, Tugwell P, Moher D, O'Neill J, Waters E, White $H$, the P-EBg: PRISMA-equity 2012 extension: reporting guidelines for systematic reviews with a focus on health equity. PLOS Med 2012, 9:e1001333.

58. Armijo-Olivo S, Stiles CR, Hagen NA, Biondo PD, Cummings GG: Assessment of study quality for systematic reviews: a comparison of the cochrane collaboration risk of bias tool and the effective public health practice project quality assessment tool: methodological research. J Eval Clin Pract 2012, 18:12-18.

59. Wallace A, Croucher K, Quilagars D, Baldwin S: Meeting the challenge: developing systematic reviewing in social policy. Policy Politics 2004, 32:455-470.

60. Popay J, Roberts H, Sowden A, Petticrew M, Arai L, Rodgers M, Britten N, Roen K, Duffy S: Guidance on the conduct of narrative synthesis in systematic reviews. London: Economic and Social Research Council (Methods Programme); 2006.

61. Higgins JPT, Green S: Cochrane handbook for systematic reviews of interventions. London: Wiley-Blackwell; 2011.

62. Campbell R, Pound P, Pope C, Britten N, Pill R, Morgan M, Donovan J: Evaluating meta-ethnography: a synthesis of qualitative research on lay experiences of diabetes and diabetes care. Soc Sci Med 2003, 56:671-684.

63. Garside R, Britten N, Stein K: The experience of heavy menstrual bleeding: a systematic review and meta-ethnography of qualitative studies. J Adv Nurs 2008, 63:550-562.

doi:10.1186/2047-2382-3-20

Cite this article as: Lovell et al:: What are the health and well-being impacts of community gardening for adults and children: a mixed method systematic review protocol. Environmental Evidence 2014 3:20.

\section{Submit your next manuscript to BioMed Central and take full advantage of:}

- Convenient online submission

- Thorough peer review

- No space constraints or color figure charges

- Immediate publication on acceptance

- Inclusion in PubMed, CAS, Scopus and Google Scholar

- Research which is freely available for redistribution 